\title{
Implementation of Fuzzy Logic for the High-Resolution Remote Sensing Images with Improved Accuracy
}

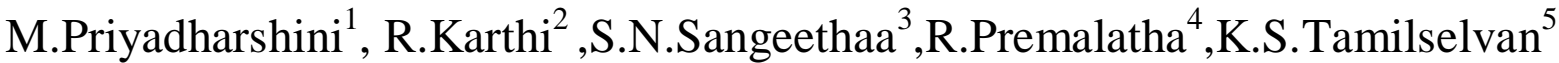 \\ ${ }^{I} P G$ Scholar., Dept. of Electronics and Communication Engineering, Velalar College of Engineering and \\ Technology., ${ }^{2} P$ GSCholar., Dept. of Electronics and Communication Engineering, Velalar College of \\ Engineering and Technology., ${ }^{3}$ Asst.Professor., Dept. of Electronics and Communication Engineering., Velalar \\ College of Engineering and Technology.
}

\begin{abstract}
In this project, an object-based approach for urban land covers classification from high resolution satellite image using Fuzzy logic. OBIA analysis of image segmentation that is, clustering of pixels into homogenous objects, and subsequent classification or labelling of the objects, and modelling based on the characteristics of objects is done using MATLAB GUI model. When applied to a satellite image, the fuzzy logic approach involves three steps. First, a membership function is computed for each pixel and each class. This membership function expresses the likelihood that the measured radiance belongs to a class with a known reflectance distribution. Thus, for each pixel, class memberships are assigned to the predetermined classes on the basis of the derived membership functions. Second, three variables are retrieved from each of the classspecific algorithms for which the pixel has membership. Third, the class memberships are used to weight the class-specific retrievals to obtain a final blended retrieval for each pixel. The algorithm was implemented in MATLAB GUI model and was tested on remotely sensed images of different sensors, resolutions and complexity levels.
\end{abstract}

Keywords - GUI (graphic user interface), object based approach, high resolution satellite image.

\section{Introduction}

Image classification, which can be defined as identification of objects in a scene captured by a vision system, is one of the important tasks of a robotic system. On the one side, the procedure of accurate object identification is known to be more difficult for computers than for people. On the other side, recently developed image acquisition systems (for instance, radar, lidar, and hyper spectral imaging technologies) capture more data from the image scene than a human vision system. Therefore, efficient processing systems must be developed in order to use these data for accurate image classification.

A Fuzzy system is a fuzzy logic based system. In this system fuzzy logic can be a basis for the representation of the various kinds of knowledge or it can model the interactions and relationships among the system variables. Fuzzy logic provides innovative tools to handle the complex and ill-defined systems where classical tools become unsuccessful. Fuzzy systems are universal approximates of non linear functions. Two aspects are important in fuzzy system one generating the best rule set and second tuning the membership functions. These should relate properly the independent and dependent variable.

Color segmentation of image is a crucial operation in image analysis and in much compute vision, image interpretation, and pattern and industrial fields such as medicine, Remote Sensing, Microscopy, content based image and video retrieval, document analysis, industrial automation and quality control. The performance of color segmentation may significantly affect the quality of an image understanding system. The most common features used in image segmentation include texture, shape, grey level intensity, and color. Probabilistic latent semantic analysis

(PLSA) [2] and latent Dirichlet allocation (LDA) [3], which were originally developed for topic discovery in text domain, are competent to accomplish this kind of work. According to the topic models, features are modeled as "visual words" by vector quantization, and images are regarded as documents and modeled as mixture of latent topics. The classification or segmentation results derived from them can be more reliant on content coherence. In addition, the effective computation based on approximate inference methods can map the high dimensional feature counts into low-dimensional topic vectors, which makes topic models remarkable. It has shown that representing images by topic mixtures outperforms using low-level features [4]. It has been reported that the annotation performance of large satellite images can benefit from topic models [5].

Despite the successful application and impressive performance of topic models, they suffer from poor spatial coherence because of the independence assumption of visual words images. A random field model such as the Markov random field (MRF) has been employed for solving this problem since the spatial information between neighboring regions in an image is thereby enforced. In [6] and [7], the authors have defined an MRF over hidden topic assignment, which has been obtained by PLSA, to describe the spatial relationship of latent 
topic,the experimental results of supervised and weakly supervised settings demonstrate that the two combined models are complementary and the segmentation and recognition accuracy is obviously improved. However, the aforementioned method was previously proposed for supervised image classification. Furthermore, the number of classes, which has a significant influence over classification result, is usually specified based on a prior or educated assignment in most classification algorithms. Moreover, because of the high resolution, satellite images have relatively richer information.

The image semantics of large structure cannot be represented properly by only using topic models. In this letter, we extend the combination of topic models and MRFs to the topic of unsupervised satellite image classification, more precisely, employing an MRF prior over latent topic labels, which are obtained by LDA, to enhance the spatial coherence information. Furthermore, the label cost mentioned in [8], which has an excellent generalization and can give its own contribution to image segmentation, is added to the random fields of latent topics. Automatically segmenting an image into coherent parts is always an important issue in segmentation. The minimum description length criterion was first proposed in [9] for unsupervised segmentation, to represent the image more compactly.

In [8], the authors have pointed out that using $\alpha$ expansion can be more powerful, because the segmentation based on such an algorithm relies on contour evolution and explicit merging of adjacent regions. Based on label cost and Bayesian information criterion (BIC), we introduce an iterative algorithm over latent topics, through which the number of classes is eventually appropriately converged. The whole process works automatically, instead of assuming it beforehand as a constant. Meanwhile, because of the global coupling function of label cost, both the consistency of semantics is well kept and the over smooth effect is avoided. Moreover, the efficiency of semantics extraction is also thereby demonstrated. The rest of this letter is organized as follows. In Section II, we review previous and related works. In Section III, we describe our approach in detail. In Section IV, we describe our result and discussion. Finally, future work in Section V.

\section{A. LDA-MRF MODEL}

\section{Previous Work}

LDA-MRF combination model (latent Dirichlet allocation-Markov Random field) is proposed to obtain accurate semantic segmentation for high resolution satellite image.

\section{B. ENERGY OPTIMIZATION}

Combining topic models with MRF is able to compensate the loss of contextual information. Local coupling is not able to keep the consistency of semantic by large structure in satellite images, because the influence on value of smooth factor in MRF leads to either over segment or over smooth.In order to balance such problem, the $\alpha$-expansion algorithm has been extended to incorporate label costs at each expansion; by doing so, the energy has been represented in the form of the sum of three terms.

$$
E(f)=\sum_{p \in P} D_{p}\left(f_{p}\right)+\sum_{p q \in \mathbb{N}} V_{P q}\left(f_{p}, f_{q}\right)+\sum_{L_{\underline{Q}} \mathbb{D}} h_{\mathrm{L}} . \delta(f) \text {. }
$$

Smooth cost $=\sum_{p q \in N} V_{P q}\left(f_{p \cdot} f_{q}\right)$

Data cost $\quad=\sum_{p \in P} D_{p}\left(f_{p}\right)$

Label cost $=\Sigma_{\underline{L} \underline{I}} h_{L} \delta(f)$

Here, the indicator function is defined on label subset L.

$$
\delta_{(f)}=\left\{\begin{array}{l}
1, \exists: f_{p} \in L \\
0, \text { otherwise }
\end{array}\right.
$$

This extended algorithm can simultaneously optimize "smooth cost" and "label cost," from which the approximate solution can be kept by a certain level. The authors in [7] have discussed that some information criteria have been employed by selection of statistical model, in order to avoid over fitting and determine the order of the model through several iterations. Since the information criteria prefer to explain the data with fewer and simpler models, they penalize the overly complex models [9].

The label costs could be scaled in some proportion (e.g., linear) to the estimated number of observations per model under the suggestion of BIC. The number of models can be determined under this algorithm by examining iterative convergence. In previous approach, label cost is added into label optimization of the latent topic fields. At the same time, combining label cost with BIC, the number of segments in the image is automatically determined by iterative operation. In this way, our method not only can add the global information into the topic model but also can automatically obtain the number of segments.

The process of iterations is as follows 
1) Using MRF spatial prior to constrain the latent topics, which are obtained by LDA, while setting the initialized number of latent topics (segments).

2) Optimizing the aforementioned latent topic fields with label cost. During the optimization, figure out whether the resulting fields match with BIC according to (1). If the minimum is reached, go to step 3); if not, i.e., the number of latent topics (segments) is still reducing, then the iteration is continued.

3) Employing the resulting number of latent topics (segments), which is finally determined in step 2), and then using the energy minimization algorithm to infer eventual labels.

\section{A. DATA SELECTION}

\section{Our Approach}

The image chosen in this project is a high resolution satellite image. The image contains three layers (RGB). It has covered a large area.

\section{B. OBJECT-ORIENTED CLASSIFICATION (FUZZY BASED) 1) Fuzzy Logic}

Object-oriented classification is mainly based upon fuzzy logic - a mathematical technique of quantifying uncertainty of statements. Fuzzy logic seeks to replace the two strictly logical statements "yes" and "no" by the continuous range of values from 0 to 1 , where 0 means "absolutely no" and 1 means "absolutely yes." Values between 0 and 1 represent various states of certainty between "no" and "yes." This approach emulates human thinking and takes into account even linguistic rules.

Fuzzy rules are "if - then" rules. If a condition is fulfilled, an action takes place. The concept behind object-oriented classification is that important semantic information necessary to interpret an image is not represented in individual pixels, but in meaningful image objects and their mutual relationships. The basic difference, especially when compared to pixel-based procedures, is that object based classification does not classify single pixels but rather image objects which are extracted through a segmentation procedure [12].

\section{Basic Procedure}

Object-based classification is composed of three basic procedures: image segmentation, object metric extraction, and classification [13]. Segmentation of an image divides it into a network of homogeneous regions at any chosen resolution. These image object primitives represent image information in an abstracted form, serving as building blocks and information carriers for subsequent classification.

Image segmentation methods fall into two main domains: knowledge driven methods (top-down) and data driven methods (bottom-up). In the top-down approach the user already knows what he wants to extract from the image, but he does not know how to perform the extraction. The system tries to find the best processing method to extract the objects by formulating a model of the desired objects. The formulated object models give the object an implicit meaning. In the bottom-up approach the segments are generated on the basis of a set of parameters and statistical methods for processing the whole image [12].

\section{2) CLUSTERING ALGORITHM}

There are many methods of clustering developed for a wide variety of purposes. Clustering algorithms used for unsupervised classification of remote sensing data vary according to the efficiency with which clustering takes place.

\section{$\mathrm{K}$ means clustering}

$\mathrm{K}$-means is the clustering algorithm used to determine the natural spectral groupings present in a data set. This accepts from analyst the number of clusters to be located in the data. The algorithm then arbitrarily seeds or locates, that number of cluster centers in multidimensional measurement space. Each pixel in the image is then assigned to the cluster whose arbitrary mean vector is closest. The procedure continues until there is no significant change in the location of class mean vectors between successive iterations of the algorithms. As Kmeans approach is iterative, it is computationally intensive and hence applied only to image subareas rather than to full scenes and can be treated as unsupervised training areas.

$\mathrm{K}$-means is one of the simplest unsupervised learning algorithms that solve the well known clustering problem. The procedure follows a simple and easy way to classify a given data set through a certain number of clusters (assume $\mathrm{k}$ clusters) fixed a priori. The main idea is to define $\mathrm{k}$ centroids, one for each cluster. These centroids should be placed in a cunning way because of different location causes different result. So, the better choice is to place them as much as possible far away from each other. The next step is to take each point belonging to a given data set and associate it to the nearest centroids. When no point is pending, the first step is completed and an early group age is done. At this point we need to re-calculate $\mathrm{k}$ new centroids as barycenters of the clusters resulting from the previous step. After we have these k new centroids, a new binding has to be 
done between the same data set points and the nearest new centroid. A loop has been generated. As a result of this loop we may notice that the $\mathrm{k}$ centroids change their location step by step until no more changes are done. In other words centroids do not move any more. Finally, this algorithm aims at minimizing an objective function, in this case a squared error function.

The objective function,

$$
\mathrm{J}=\sum_{\mathrm{j}=1}^{\mathrm{k}} \sum_{\mathrm{i}=1}^{\mathrm{x}}\left\|\mathrm{x}_{\mathrm{i}}{ }^{(\mathrm{j})}-\mathrm{cj}\right\|^{2}
$$

Clustering is a way to separate groups of objects. K-means clustering treats each object as having a location in space. It finds partitions such that objects within each cluster are as close to each other as possible, and as far from objects in other clusters as possible. K-means clustering requires that you specify the number of clusters to be partitioned and a distance metric to quantify how close two objects are to each other. Since the color information exists in the ' $a * b^{* '}$ space, your objects are pixels with ' $a{ }^{* '}$ and ' $b{ }^{* '}$ Euclidean distance metric.

\section{ISODATA clustering}

K-mean and the ISODATA clustering algorithms are iterative procedures. In general, both of them assign first an arbitrary initial cluster vector. The second step classifies each pixel to the closest cluster. In the third step the new cluster mean vectors are calculated based on all the pixels in one cluster. The second and third steps are repeated until the "change" between the iteration is small. The "change" can be defined in several different ways, either by measuring the distances the mean cluster vector have changed from one iteration to another or by the percentage of pixels that have changed between iterations. The ISODATA algorithm has some further refinements by splitting and merging of clusters. Clusters are merged if either the number of members (pixel) in a cluster is less than a certain threshold or if the centers of two clusters are closer than a certain threshold. Clusters are split into two different clusters if the cluster standard deviation exceeds a predefined value and the number of members (pixels) is twice the threshold for the minimum number of members.

The ISODATA algorithm is similar to the k-means algorithm with the distinct difference that the ISODATA algorithm allows for different number of clusters while the k-means assumes that the number of clusters is known a priori.

The objective of the k-means algorithm is to minimize the within cluster variability. The objective function (which is to be minimized) is the sums of squares distances (errors) between each pixel and its assigned cluster center.

$$
S S_{\text {distances }}=\Sigma_{\forall x}[x-c(x)]^{2}
$$

Where $C(x)$ is the mean of the cluster that pixel $x$ is assigned to.

Minimizing the $S S_{\text {distances }}$ is equivalent to minimizing the Mean Squared Error (MSE). The MSE is a measure of the within cluster variability.

$$
\mathrm{MSE}=\frac{\sum_{\forall x}[x-c(x)]^{2}}{(N-C) b}=\frac{s s_{\text {distances }}}{(N-C) b}
$$

Where $\mathrm{N}$ is the number of pixels, $\mathrm{c}$ indicates the number of clusters, and $\mathrm{b}$ is the number of spectral bands.

\section{Result And Discussion}

The various experiment carried out in MATLAB v7.5.0 (R2007b).

TABLE I

EXPERIMENTAL RESULTS OF ENTROPY

\begin{tabular}{|c|c|c|}
\hline image & algorithm & Entropy \\
\hline \multirow{2}{*}{ Image 1 } & Algorithm (I) & 12.44508 \\
\cline { 2 - 3 } & Algorithm (II) & 7.55171 \\
\hline \multirow{2}{*}{ Image 2 } & Algorithm (I) & 12.44508 \\
\cline { 2 - 3 } & Algorithm (II) & 8.2281 \\
\hline
\end{tabular}

LDA-MRF method, $k$-means clustering, iterative self-organizing data analysis technique (ISODATA) clustering and Object-Oriented Classification (Fuzzy Based) are examined .On the one hand, the qualitative evaluation is examined in terms of the semantics of the segmentation results. On the other hand, the results are also quantitatively evaluated in terms of the purity and entropy. 


\section{Future Work}

In future the utilization of K-means clustering and fuzzy based object-orientation classification model for unsupervised satellite image classification will be designed so as to improve the classification accuracy. While the above two results presented here by using isodata clustering and LDA-MRF are encouraging, there is still a need for further improvements. Future extensions could include many algorithms related to fuzzy based classification.
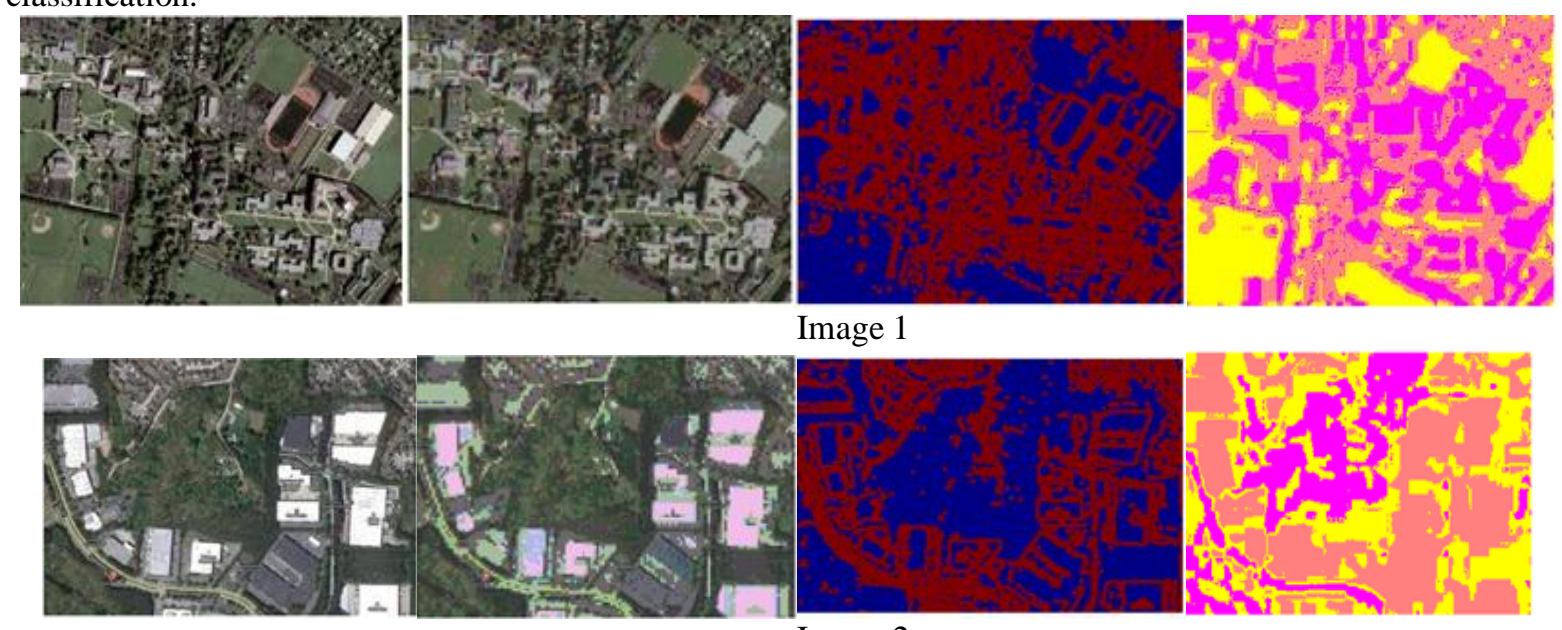

Image 1

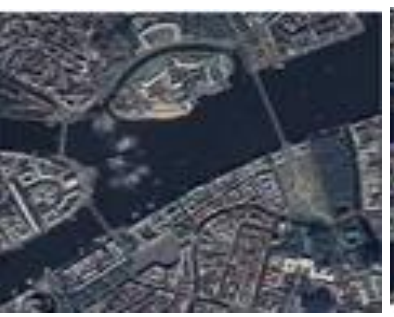

Image 3

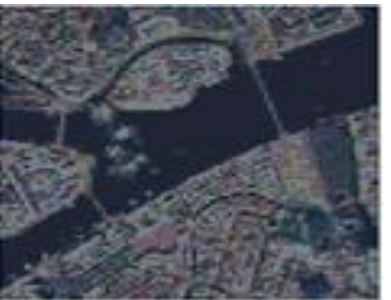

Ground-Truth

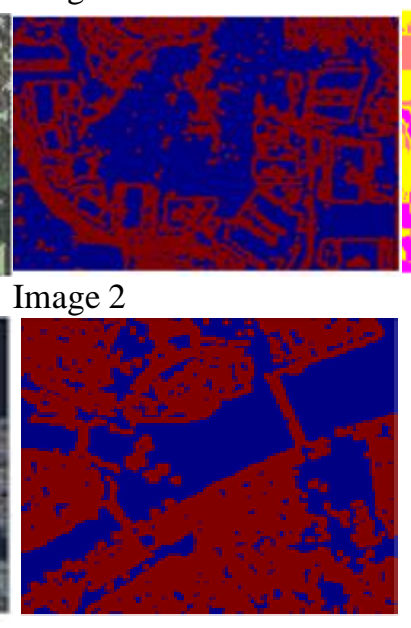

LDA-MRF
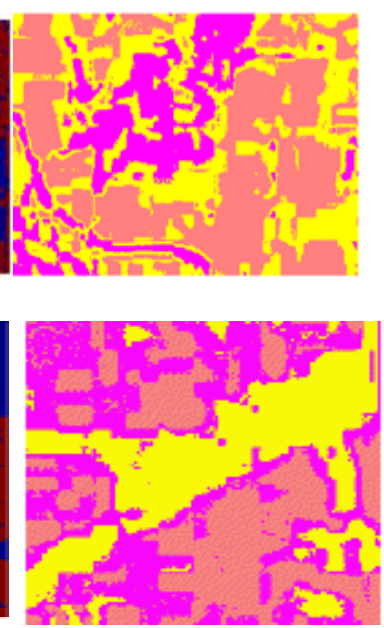

ISODATA

Fig. 1. Classification results of three different scenes. The first column shows the input images, second column shows the ground truth images and the third columns shows the classification results of LDA-MRF method, and finally classification results of ISODATA clustering is displayed.

By comparing the above methods better classification results are examined.

\section{References}

[1] KanXu,Wen Yang, Gang Liu, and Hong Sun, (2013) "Unsupervised Satellite Image Classification Using Markov Field Topic Model".

[2] Delong. A, Osokin.A, Isack H.N, and Boykov.Y,(2010) "Fast approximate energy minimization with label costs," in Proc. IEEE Conf. Comput. Vis. Pattern Recog., pp. 2173-2180.

[3] Liénou.M, Maitre.H, and Datcu.M, (2010), "Semantic annotation of satellite images using latent Dirichlet allocation," IEEE Geosci. Remote Sens. Lett, vol. 7, no. 1, pp. 28-32.

[4] Larlus.D and Jurie.F, Apr.(2009) "Latent mixture vocabularies for object categorization and segmentation," Image Vis. Comput., vol. 27 , no. 5 , pp. 523-534

[5] MacKay.D.J.C,(2003)"Information Theory, Inference, and Learning Algorithms", vol. 8. Cambridge, U.K.: Cambridge Univ.,p. 12.

[6] Memarsadeghi.N,Mount.D,Netanyahu.N.S, Le Moigne.J, and de Berg.M,(2007) "A fast implementation of the ISODATA clustering algorithm," Int. J. Comput. Geom. Appl., vol. 17, no. 1, pp. 71-103.

[7] Rosenberg and J. Hirschberg, "V-measure: A conditional entropy based external cluster evaluation measure," in Proc. Joint conf.EMNLPCONLL, 2007,pp. 410-420.

[8] Tang. W. Yi, H, and Chen.Y, “An object-oriented semantic clustering algorithm for high-resolution remote sensing images using the aspect model "May (2011) IEEE Geosci. Remote Sens. Lett., vol. 8, no. 3, pp. 522-526.

[9] Verbeek.J and Triggs.B, "Region classification with Markov field aspect models," (2007) in Proc. IEEE Conf. Comput. Vis. Pattern Recog., pp. 1-8.

[10] Yang.W, Dai.D, Wu.J, and He.C, "Weakly supervised polarimetric SAR image classification with multi-modal Markov aspect model," (2010) in Proc. ISPRS, TC VII Symp. (Part B), 100 Years ISPRSAdvancing Remote Sensing Science, Vienna, Austria, Jul. 5-7, pp. 669-673.

[11] Zhu.S.C and Yuille.A.L, Sep(1996) "Region competition: Unifying, snakes, region growing, and Bayes/MDL for multiband image, segmentation,” IEEE Trans. Pattern Anal. Mach. Intell., vol. 18, no. 9, pp. 884-900.

[12] Civanlar,R.,Trussel,H.,(1986)Constructing membership functions using statistical data. IEEE Fuzzy Sets and Systems 18, $1-14$. . Curlander, J., Kober, W., 1992.

[13] Proc.IGARSS . IEEE Press, New York, pp. 854- 856."Rule based system for thematic classification in SAR imagery". 